\title{
Impedance spectroscopy study of the electrical properties of composites of $\mathrm{CaCu}_{3} \mathrm{Ti}_{4} \mathrm{O}_{12}-\mathrm{CuO}$
}

\author{
N.A. Sekushin ${ }^{1}$, N. A. Zhuk ${ }^{2, \dagger}$, L. A. Koksharova², V. A. Belyy ${ }^{1}$, B. A. Makeev³, \\ D. S. Beznosikov ${ }^{2}$, M. V. Yermolina ${ }^{4}$ \\ †nzhuck@mail.ru
}
${ }^{1}$ Institute of Chemistry of Komi Scientific Center, Ural Branch of RAS, 48 Pervomaiskaya St., Syktyvkar, 167982, Russia ${ }^{2}$ Syktyvkar State University, 55 Oktyabrsky Av., Syktyvkar, 167001, Russia
${ }^{3}$ Institute of Geology of Komi Scientific Center, Ural Branch of RAS, 54 Pervomaiskaya St., Syktyvkar, 167982, Russia ${ }^{4}$ University of Illinois Chicago, 845 W. Taylor St., MC 111, Chicago, IL, 60607, United States

Calcium copper titanate $\mathrm{CaCu}_{3} \mathrm{Ti}_{4} \mathrm{O}_{12}$ attracts considerable attention of researchers due to the manifestation of extremely high values of dielectric constant $\left(\varepsilon \sim 10^{4}-10^{5}\right)$ in wide temperature $(100-600 \mathrm{~K}$ ) and frequency (from $20 \mathrm{~Hz}$ to $1 \mathrm{MHz}$ ) ranges. In spite of the active studies of $\mathrm{CaCu}_{3} \mathrm{Ti}_{4} \mathrm{O}_{12}$, there are unresolved questions on the influence of microstructure, the presence of copper (II) oxide layers in the intergrain space of the ceramics and on the selective influence of paramagnetic dopants on the electrophysical properties of the compound. In this connection, the electrical properties of the $\mathrm{CaCu}_{3} \mathrm{Ti}_{4} \mathrm{O}_{12}-\mathrm{CuO}_{\mathrm{O}}$ composite were studied by the method of impedance spectroscopy. The composite was synthesized by the solid-phase method of staged calcination at $650^{\circ} \mathrm{C}, 850^{\circ} \mathrm{C}$ and $1050^{\circ} \mathrm{C}$ for 50 hours. The analysis of the impedance spectroscopy results has shown that $\mathrm{CaCu}_{3} \mathrm{Ti}_{4} \mathrm{O}_{12}-\mathrm{CuO}$ is a material with mixed electron-ion conductivity. The strong dispersion of the conductivity at room temperature was explained by the polarization of copper ions or protons, which can be present in the sample due to the dissociative adsorption of water molecules from the air. The minimum of the dispersion of the dielectric constant was found at a temperature of about $300^{\circ} \mathrm{C}$, which was explained by the appearance of oxygen conductivity substantially neutralizing the cation conductivity. The simulation of electrical properties by the method of constructing equivalent circuits showed that the sample consisted of two layers. The high-resistivity layer was assigned to the crystalline part of the sample, and the lowresistivity layer to the intergranular part. The significant change in the electrical properties of the sample at the temperature of $200-225^{\circ} \mathrm{C}$ is not associated with the occurrence of a phase transition in it.

Keywords: calcium copper titanate, composite, impedance spectroscopy, electrical properties.

УДК: $537.3,537.9,538.9$

\section{Исследование методом импеданс-спектроскопии электрических свойств композита $\mathrm{CaCu}_{3} \mathrm{Ti}_{4} \mathrm{O}_{12}-\mathrm{CuO}$}

Секушин $^{1}$ Н.А., Жук ${ }^{2 \dagger}$ Н. А., Кокшарова ${ }^{2}$ Л. А., Белый ${ }^{1}$ В. А., Макеев ${ }^{3}$ Б. А., Безносиков 2 Д. С., Ермолина ${ }^{4}$ М. В.

†nzhuck@mail.ru

\author{
${ }^{1}$ Институт химии Коми НЦ УрО РАН, Первомайская ул., 48, Сыктывкар, 167982, Россия \\ ${ }^{2}$ Сыктывкарский государственный университет, Октябрьский проспект, 55, Сыктывкар, 167001, Россия \\ ${ }^{3}$ Институт геологии Коми НЦ УрО РАН, Первомайская ул., 54, Сыктывкар, 167982, Россия \\ ${ }^{4}$ University of Illinois Chicago, 845 W. Taylor St., MC 111, Chicago, IL, 60607, USA
}

Титанат кальция-меди $\mathrm{CaCu}_{3} \mathrm{Ti}_{4} \mathrm{O}_{12}$ привлекает пристальное внимание ученых в связи с проявлением огромных значений диэлектрической проницаемости $\left(\varepsilon \sim 10^{4}-10^{5}\right)$ в широком температурном $(100-600$ К) и частотном (от 20 Гц до 1 МГц) интервалах. Несмотря на активные исследования $\mathrm{CaCu}_{3} \mathrm{Ti}_{4} \mathrm{O}_{12}$ до сих пор остаются не разрешенными вопросы, связанные с влиянием микроструктуры керамики, присутствием в межзеренном пространстве керамики 
прослоек из оксида меди (II), избирательным воздействием парамагнитных допантов на электрофизические свойства соединения. В связи с этим выполнено исследование методом импеданс-спектроскопии электрических свойств композита $\mathrm{CaCu}_{3} \mathrm{Ti}_{4} \mathrm{O}_{12}-\mathrm{CuO}$, синтезированного твердофазным методом путем поэтапного прокаливания при температурах $650^{\circ} \mathrm{C}, 850^{\circ} \mathrm{C}, 1050^{\circ} \mathrm{C}$ в течение 50 часов. На основании анализа данных, полученных методом импедансспектроскопии, показано, что $\mathrm{CaCu}_{3} \mathrm{Ti}_{4} \mathrm{O}_{12}-\mathrm{CuO}$ является материалом со смешанной электронно-ионной проводимостью. Сильная дисперсия проводимости при комнатной температуре объяснена участием в поляризационном процесс ионов меди или протонов, которые могут присутствовать в образце вследствие диссоциативной адсорбции молекул воды из воздуха. При температуре около $300^{\circ} \mathrm{C}$ обнаружен минимум дисперсии диэлектрической проницаемости, что объяснено появлением кислородной проводимости, которая в значительной степени нейтрализует катионную проводимость. Моделирование электрических свойств методом построения эквивалентных схем показало, что образец можно рассматривать состоящим из двух слоев. Высокоомный слой был отнесен к кристаллической части образца, а низкоомный - к межзеренной прослойке. Установлено, что существенное изменение электрических свойств композита титаната кальция-меди при температуре $200-225^{\circ} \mathrm{C}$ не связано с протеканием фазового перехода в нем.

Ключевые слова: титанат кальция-меди, композит, импеданс-спектроскопия, электрические свойства.

\section{1. Введение}

Среди материалов, обладающими огромными значениями диэлектрической проницаемости $\left(\varepsilon \sim 10^{4}-10^{5}\right)$ выделяется титанат кальция-меди $\mathrm{CaCu}_{3} \mathrm{Ti}_{4} \mathrm{O}_{12}$ с кубической структурой двойного перовскита (Im3) [1,2], первые сведения о котором получены в 1967 г. из работы [3], а открытие уникальных диэлектрические свойств этого соединения принадлежит М. Субраманиану с соавторами [4]. Значительным преимуществом данного соединения является сохранение высоких значений диэлектрической проницаемости в широком интервале температур $100-600$ К в диапазоне частот от 20 Гц до 1 МГц [5-7]. Диэлектрическая проницаемость $\mathrm{CaCu}_{3} \mathrm{Ti}_{4} \mathrm{O}_{12}$ характеризуется частотной дисперсией и при температуре ниже $100 \mathrm{~K}$ резко понижается без каких-либо структурных изменений [8-12]. Такое поведение титаната кальция-меди обуславливает значительный интерес исследователей как с прикладной, так и фундаментальной точек зрения [12-18]. В настоящее время существует множество моделей, объясняющих аномальное диэлектрическое поведение титаната кальция-меди. Преимущественной среди них является модель, согласно которой микроструктура соединения рассматривается как зерно-прослойка, формирующая потенциальный барьер на границах зерен или доменов и ответственная за диэлектрические свойства соединения [19-21]. Несмотря на активные исследования титаната кальция-меди, ряд вопросов остаются не вполне изученными. В последнее время в некоторых работах отмечается $[9,22]$, что фазовый состав и микроструктура титаната кальция-меди чутко реагируют на условия синтеза - атмосферу, температуру и длительность термообработки. Нередко, наряду с титанатом кальция-меди при твердофазном синтезе образуется оксид меди (II), заполняющий межзеренное пространство керамики и оказывающий влияние на функциональные свойства материала $[9,17]$. В связи с этим, исследование влияния примесей, в частности, оксида меди (II) на электрические свойства титаната кальция-меди представляется особо актуальным. В связи с этим, в настоящей работе представлены результаты исследования методом импеданс-спектроскопии электрических свойств композита $\mathrm{CaCu}_{3} \mathrm{Ti}_{4} \mathrm{O}_{12}-\mathrm{CuO}$.

\section{2. Экспериментальная часть}

Синтез титаната кальция меди проведен стандартным керамическим методом из оксидов меди (II), титана (IV) и карбоната кальция квалификации «ос.ч.» при температурах обжига $650^{\circ} \mathrm{C}, 850^{\circ} \mathrm{C}$ и $1050^{\circ} \mathrm{C}$ в течение 50 часов. Образцы изготовлены в форме диска диаметром $\sim 13$ мм, толщиной $\sim 3$ мм. Для исследования электрических свойств композита на обе стороны диска нанесен токопроводящий слой методом вжигания серебряной пасты. Фазовый состав препаратов контролировали методами электронной сканирующей микроскопии (электронный сканирующий микроскоп Tescan VEGA 3LMN, энергодисперсионный спектрометр INCA Energy 450) и рентгенофазового анализа (ДРОН-4-13, $\mathrm{Cu}_{\mathrm{Ka}}$-излучение), параметры элементарной ячейки твердых растворов рассчитаны с использованием пакета программ CSD [23]. Измерение электрических свойств $\mathrm{CaCu}_{3} \mathrm{Ti}_{4} \mathrm{O}_{12}-\mathrm{CuO}$ при комнатной температуре выполнено с помощью анализатора иммитанса широкополосного Е7-28 (ОАО “МНИПИ”, республика Беларусь). Исследование образцов в области высоких температур проведено с помощью импедансметра Z-1000P (Elins, г. Черноголовка Московской обл.). Частотные зависимости измеряли в диапазоне от 100 Гц до 1 МГц. При частотах менее 100 Гц исследование было затруднено из-за сильных шумов, а при частотах более 1 МГц наблюдалась систематическая ошибка измерений из-за импеданса подводящих проводов. Предварительные исследования показали, что образцы $\mathrm{CaCu}_{3} \mathrm{Ti}_{4} \mathrm{O}_{12}-\mathrm{CuO}$ имеют линейные вольтамперные характеристики по крайней мере до входного напряжения 2 В. Это позволило подавать на образец максимально возможный для Z-1000Р гармонический сигнал амплитудой 250 мВ, что позволяло снизить уровень шума. При измерении частотных зависимостей было запрограммировано 6 точек на каждой декаде частоты. Окончательное значение импеданс в каждой точке определялось усреднением по 100 измерениям. Термический анализ образцов проведен в температурном интервале $23 \div 1100^{\circ} \mathrm{C}$ при использовании анализатора STA 409 PC Luxx (Germany). 


\section{3. Результаты и их обсуждение}

Поданным рентгенофазового анализа иэлектронной сканирующей микроскопии получен композит $\mathrm{CaCu}_{3} \mathrm{Ti}_{4} \mathrm{O}_{12}$ с межзеренными включениями-прослойками оксида меди (Рис. 1a, b), связность компонентов в котором относится к типу 3-1 по классификации Ньюнхэма [24]. На рентгенограмме наблюдается наложение рефлексов от $\mathrm{CaCu}_{3} \mathrm{Ti}_{4} \mathrm{O}_{12}$ и примесных фаз $-\mathrm{CaTiO}_{3}$ и оксида меди (II) (Рис. S1, дополнительный материал).

Приближенная оценка содержания оксида меди в керамике по данным рентгеновской дифракции и дифференциально-сканирующей калориметрии (Рис. S2, дополнительный материал) показала, что оксида меди в образце титаната кальция-меди не более 3 - 5 мольных процента от общего содержания меди в $\mathrm{CaCu}_{3} \mathrm{Ti}_{4} \mathrm{O}_{12}$. Параметры элементарной ячейки титаната кальция-меди составляют $0.7381 \mathrm{~nm}$ (пр. группа $\operatorname{Im} 3$ ), что соответствует литературным данным $[1,3]$.

При исследовании электрических свойств данного материала при комнатной температуре были обнаружены два поляризационных процесса, которые разделены точкой перегиба на кривой $\varepsilon(\omega)$ (Рис. 2). Такой же вывод можно сделать из частотной зависимости $\operatorname{tg} \delta$. На этой кривой наблюдаются склоны двух максимумов. Оба обнаруженных процесса действуют в широком диапазоне частот. Их средние времена релаксации различаются на 6 - 7 порядков.

При повышении температуры время релаксации уменьшается, вследствие чего частотные зависимости смещаются в направлении более высоких частот. Поэтому для извлечения полной информации необходимо одновременно изучать воздействие на объект как электромагнитного поля, так и температуры. Это достигается измерением импеданса при нескольких фиксированных температурах в диапазоне от 0 до $450^{\circ} \mathrm{C}$. Результаты исследования влияния температуры на импеданс $\mathrm{CaCu}_{3} \mathrm{Ti}_{4} \mathrm{O}_{12}-\mathrm{CuO}$ представлены на Рис. 3 в координатах «удельная проводимость $(\sigma)-$ диэлектрическая проницаемость $(\varepsilon) »$. Достоинством данной системы координат является то, что весь экспериментальный материал удается разместить на одном

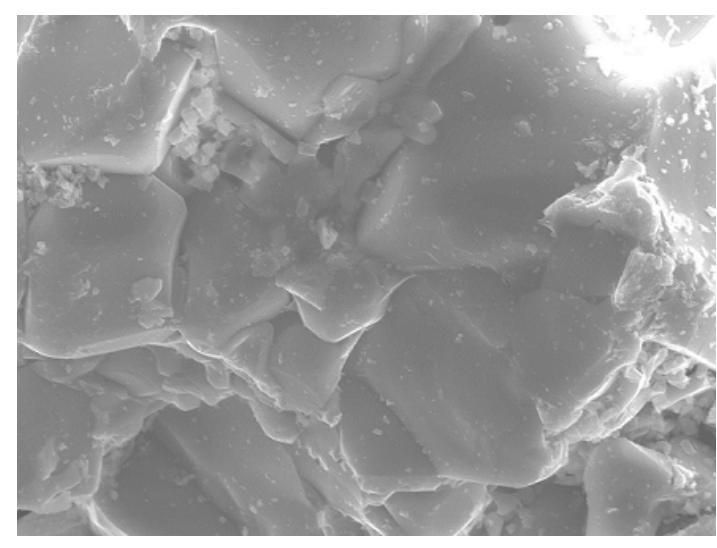

или двух графиках. На Рис. 3 изображена сетка, состоящая из изотерм $\varepsilon(\sigma, T=$ const $)$ и изочастот $\varepsilon(\sigma, \omega=$ const $)$. Переход от вещественной $\left(Z^{\prime}\right)$ и мнимой $\left(Z^{\prime \prime}\right)$ частей импеданса к координатам $(\varepsilon, \sigma)$ осуществляется с помощью следующих формул:

$$
\varepsilon=\frac{h}{\varepsilon_{0} S} \frac{-Z^{\prime \prime}}{\omega|Z|^{2}}, \quad \operatorname{tg} \delta=-\frac{Z^{\prime}}{Z^{\prime \prime}}
$$

где $h$ и $S-$ толщина и площадь поверхности образца, имеющего форму плоскопараллельной пластины или диска; $\varepsilon_{0}$ - диэлектрическая постоянная вакуума.

Представление данных импеданс-спектроскопии в координатах $(\varepsilon, \sigma)$ позволяет обнаруживать не толькофазовые переходы, но и тонкие эффекты в материалах сосмешанной электронно-ионной проводимостью $[25,26]$. Из анализа Рис. 3 можно сделать следующие выводы. При низких температурах наблюдается сильная дисперсия проводимости. Это означает, что поглощение электромагнитной энергии сильно зависит от частоты $\omega$, такой эффект наблюдаются у ионных проводников [25]. В данном случае следует ожидать высокую подвижность либо протонов, либо катионов меди. При повышении температуры при частотах $\omega<10$ кГц дисперсия сначала достигает максимума $\left(T=225^{\circ} \mathrm{C}\right)$, а затем падает до минимума $\left(T=300-325^{\circ} \mathrm{C}\right)$. При дальнейшем повышении температуры дисперсия вновь увеличивается. В последних исследованиях методом изотопного обмена кислорода было установлено, что у сложных оксидов со структурой пирохлора анионы $\mathrm{O}^{2-}$ приобретают подвижность при $T \geq 250^{\circ} \mathrm{C}$ [27]. Это явление, по-видимому, наблюдается и у композита $\mathrm{CaCu}_{3} \mathrm{Ti}_{4} \mathrm{O}_{12}-\mathrm{CuO}$. Таким образом, у исследуемых образцов существует температурная область, в которой сосуществуют ионные носители, имеющие разноименные заряды. В этом случае возможно образование анионно-катионных ассоциатов, что приводит к снижению общей концентрации носителей заряда и к уменьшению низкочастотной диэлектрической проницаемости. При более высоких температурах возможно полное подавление катионной проводимости, так как в $\mathrm{CaCu}_{3} \mathrm{Ti}_{4} \mathrm{O}_{12}$-CuO атомов кислорода содержится в 4 раза больше, чем атомов меди.

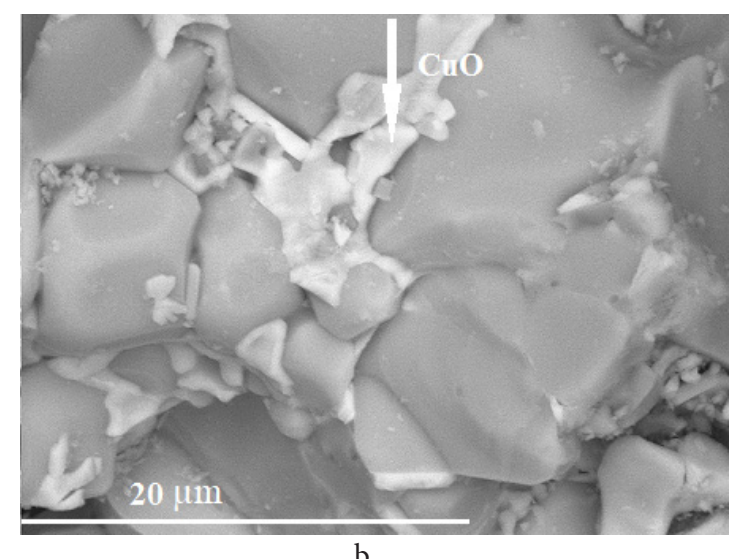

Рис. 1. Микрофотографии поверхности в режиме вторичных электронов (a), упругоотраженных (b) композита СaCu $\mathrm{Ti}_{4} \mathrm{O}_{12}-\mathrm{CuO}_{\text {. }}$ Fig. 1. Microphotographs of the surface in the regime of secondary electrons (a), elastically reflected electrons (b) of the composite $\mathrm{CaCu}_{3} \mathrm{Ti}_{4} \mathrm{O}_{12}-\mathrm{CuO}$. 


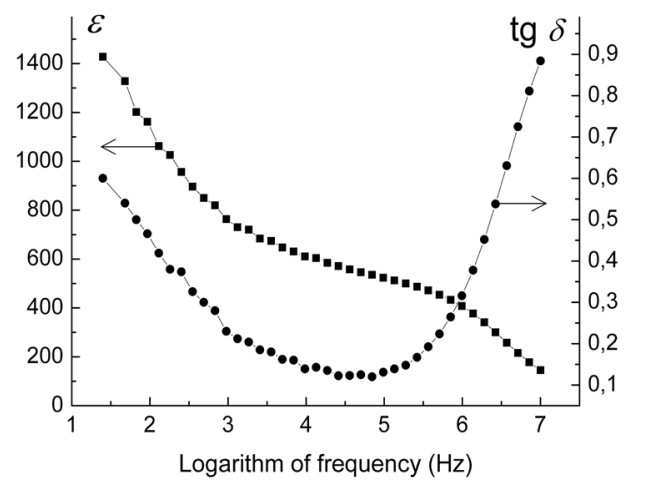

Pис. 2. Зависимости диэлектрической проницаемости $\varepsilon$ и тангенса угла диэлектрических потерь $\operatorname{tg} \delta$ образца $\mathrm{CaCu}_{3} \mathrm{Ti}_{4} \mathrm{O}_{12}-\mathrm{CuO}$ от десятичного логарифма частоты в интервале от 25 Гц до 10 МГц при температуре $T=20^{\circ} \mathrm{C}$.

Fig. 2. Dependences of the dielectric constant $\varepsilon$ and the dielectric loss tangent tg $\delta$ of the sample $\mathrm{CaCu}_{3} \mathrm{Ti}_{4} \mathrm{O}_{12}-\mathrm{CuO}$ on the common logarithm of frequency in the range from $25 \mathrm{~Hz}$ to $10 \mathrm{MHz}$ at the temperature of $T=20^{\circ} \mathrm{C}$.

Важным этапом анализа данных, полученных методом импеданс-спектроскопии, является построение эквивалентной схемы (ЭС). Эта процедура позволяет перейти от частотно-зависимых характеристик к небольшому числу не зависящих от частоты параметров. В настоящей работе построение ЭС осуществлено с помощью программы ZView (Scribner Association). Эта программа является эффективным средством анализа импеданс-спектров, поскольку позволяет подобрать наиболее точную ЭС для аппроксимации эксперимнтальных данных, рассчитывает по методу наименьших квадратов параметры ЭС, определяет среднеквадратичные отклонения и критерии точности аппроксимации. Такой анализ был выполнен для каждой температуры, что позволило построить наиболее точные ЭС (Рис. 4). В Табл. S1 (дополнительный материал) приведены параметры всех ЭС, рассчитанные программой ZView. В столбце 9 записаны значения «критерия согласия Пирсона $\chi^{2}$ » (Chi-Squared), характеризующее точность модели. При $\chi^{2}<10^{-4}$ ЭС максимально точна. Если $\chi^{2}>10^{-3}$, то отклонение экспериментальных точек от теоретической кривой можно наблюдать визуально. Следует отметить, что величина критерия Пирсона зависит не только от точности модели, но и от качества экспериментального материала, например, наличия шумов. Именно этим объясняется высокое значение $\chi^{2}$ при $T \leq 100^{\circ} \mathrm{C}$. У высокоомных образцов $\chi^{2}$ всегда больше, чем у низкоомных образцов.

В приведенных на Рис. 4 схемах использованы резисторы $(R)$, конденсаторы $(C)$ а также элементы постоянной фазы $(C P E)$, импеданс которых рассчитывают по формуле:

$$
Z_{C P E}=\frac{1}{T_{C P E}(j \omega)^{P_{C P E}}},
$$

где $P_{C P E}-$ безразмерная константа, задающая сдвиг фазы; $T_{\text {CPE }}$ - параметр, размерность которого зависит от величины $P_{C P E} ; j-$ мнимая единица.

Из Рис. 4 и Табл. S1 (дополнительный материал) следует, что структура ЭС претерпевает изменения по мере повышения температуры, сами параметры также зависят от температуры. В целом, двухзвенная структура ЭС указывает на слоистое строение композита, что согласуется с электронно-микроскопическими изображениями (Рис. 1). Звено с резистором R1 моделирует «высокоомный слой», а звено с резистором $R 3$ моделирует «низкоомный слой», что следует из данных Табл. S1 (дополнительный материал). При температурах менее $100^{\circ} \mathrm{C}$ низкоомный слой исследовать не удалось, так как его частотные характеристики оказались в зашумленном диапазоне частот менее 100 Гц. Соответствующая ЭС (Рис. 4 a) имеет только одно звено. При $T>425^{\circ} \mathrm{C}$ высокочастотная поляризация, которую моделирует звено с R1, стала настолько быстрой, что её наблюдение в доступном диапазоне частот стало невозможным. Соответствующие частотные характеристики сместились в область частот более 1 МГц. В результате ЭС образца упростилась (Рис. $4 \mathrm{~d}$ ).

Из данных Табл. S1 (дополнительный материал) можно определить некоторые характеристики упомянутых выше слоев. Прежде всего, рассмотрим зависимости проводимостей резисторов $R 1$ и $R 3$ в Аррениусовом масштабе (Рис. 5).

$\mathrm{У}$ резистора $R 1$ при $200^{\circ} \mathrm{C}$ происходит резкое изменение энергии активации с $E_{a}=0.432$ до 1.04 эВ, а у $R 3$

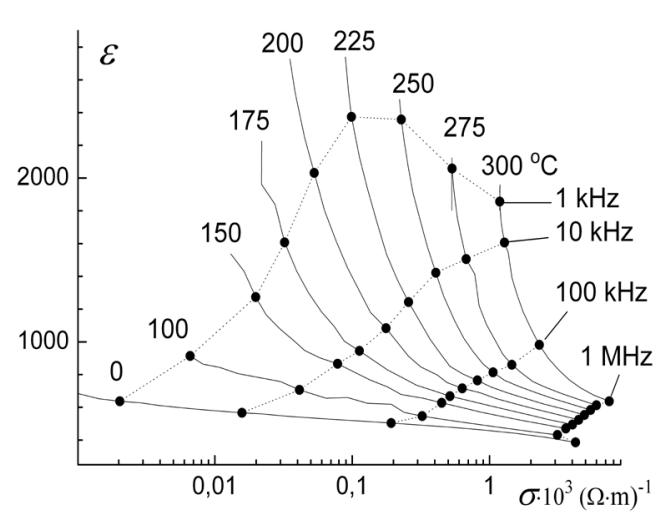

a

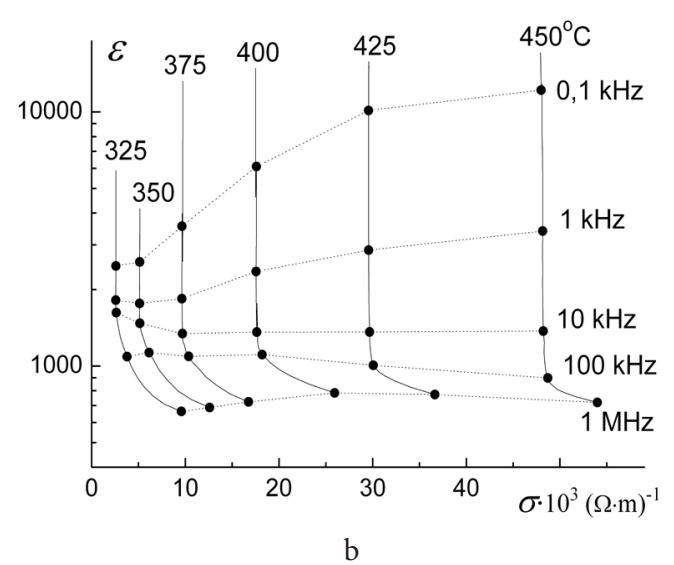

b

Рис. 3. Годографы $\mathrm{CaCu}_{3} \mathrm{Ti}_{4} \mathrm{O}_{12}$ - $\mathrm{CuO}$ в координатах «удельная проводимость - диэлектрическая проницаемость» в температурном интервале $23-300^{\circ} \mathrm{C}$ (a) и $325-450^{\circ} \mathrm{C}(\mathrm{b})$.

Fig. 3. Hodographs of $\mathrm{CaCu}_{3} \mathrm{Ti}_{4} \mathrm{O}_{12}-\mathrm{CuO}$ in the coordinates of «specific conductance - dielectric constant» in the temperature range of $23-300^{\circ} \mathrm{C}$ (a) and $325-450^{\circ} \mathrm{C}(\mathrm{b})$. 

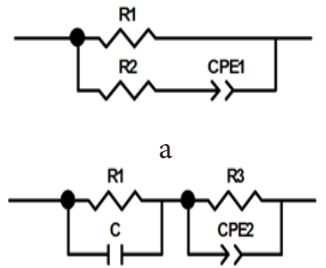

$c$

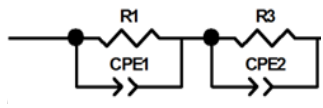

b

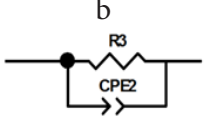

d
Pис. 4. Эквивалентные схемы композита $\mathrm{CaCu}_{3} \mathrm{Ti}_{4} \mathrm{O}_{12} \mathrm{CuO}$ для диапазона температур ( $\left.{ }^{\circ} \mathrm{C}\right): 0-100$ (a); $125-325$ (b); $350-425$ (c); 450 (d).

Fig. 4. Equivalent circuits of the composite $\mathrm{CaCu}_{3} \mathrm{Ti}_{4} \mathrm{O}_{12}-\mathrm{CuO}$ for the temperature ranges $\left({ }^{\circ} \mathrm{C}\right): 0-100(\mathrm{a}) ; 125-325(\mathrm{~b}) ; 350-425$ (c); 450 (d).

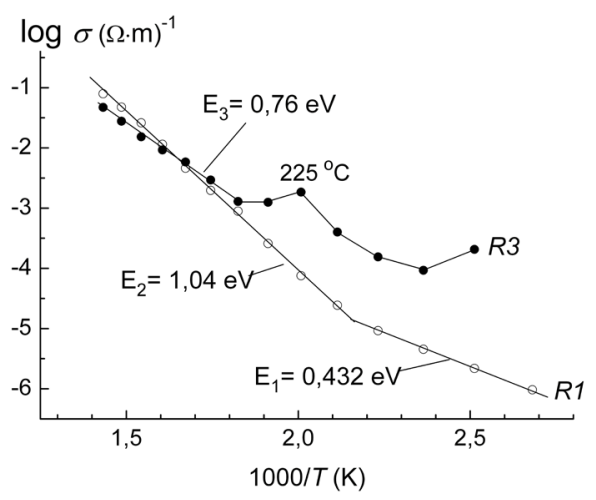

Рис. 5. Зависимости десятичного логарифма проводимости $\sigma$ резисторов $R 1$ и $R 3$ от обратной температуры.

Fig. 5. Dependences of the common logarithm of the conductivity $\sigma_{c}$ of the resistors $R 1$ and $R 3$ on the reciprocal temperature.

при $225^{\circ} \mathrm{C}$ наблюдается локальный максимум, что может быть связано с активизацией анионов кислорода. Для каждого линейного участка зависимости рассчитаны значения энергии активации (эВ): $E_{1}=0.432 \pm 0.006$; $E_{2}=1.04 \pm 0.01 ; E_{3}=0.76 \pm 0.02$. Из Рис. 5 можно заключить, что $R 1$ относится, скорее всего, к кристаллической фазе, a $R 3$ - к межзеренным слоям-включениям. Кислород в пограничных слоях, вероятно, обладает более высо-

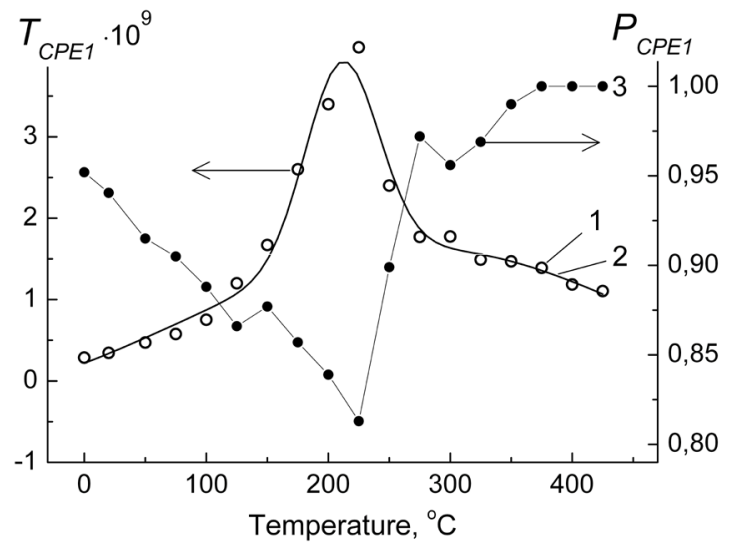

кой подвижностью, чем в объеме кристалла. В результате анализа температурных зависимостей параметров CPE1 и CPE2, прежде всего обращает внимание тот факт, что параметры этих элементов близки по величине, $T_{\text {СPE1 }}$ отличается от $T_{\text {СРE2 }}$ в 3-4 раза, а их средние значения практически совпадают (Рис. $6 \mathrm{a}, 6 \mathrm{~b}$ ), что указывает на близкие электрические свойства слоев.

Параметры $P_{C P E 1}$ и $P_{C P E 2}$ имеют понятный физический смысл, если они равны 1, то CPE превращается в идеальный конденсатор с $\operatorname{tg} \delta=0$. Такую среду можно считать максимально однородной по своим диэлектрическим характеристикам. Чем сильнее параметр $Р$ отклоняется от 1 , тем вещество становится менее однородным, если представить материал в виде совокупности электрических диполей, то неоднородность означает присутствие диполей с большим разбросом времен релаксации, такую среду можно назвать поляризационно неоднородной. Из Рис. 6 а можно сделать вывод о том, что при повышении температуры поляризационная неоднородность высокоомного слоя сначала монотонно увеличивается до $T=225^{\circ} \mathrm{C}$, а затем начинает быстро убывать, в конечном итоге $P_{C P E 1}$ становится равным 1 (кривая 3, Рис. 6а). Что касается низкоомного слоя, то $P_{P C E 2}$ при повышении температуры изменяется хаотично, при этом среднее значение $P_{\text {PCE2 }}$ меньше среднего значения $P_{P C E 1}$. Таким образом, «низкоомный слой» обладает значительным разбросом своих характеристик. Следовательно, он, по-видимому, представляет собой межзеренную прослойку. Резкое изменение некоторых электрических характеристик высокоомного слоя при $200-225^{\circ} \mathrm{C}$ может указывать на вероятность фазового перехода. Между тем, исследования термического поведения композита $\mathrm{CaCu}_{3} \mathrm{Ti}_{4} \mathrm{O}_{12}-\mathrm{CuO}$ в температурном интервале $25-1200^{\circ} \mathrm{C}$ (Рис. S1, дополнительный материал) свидетельствуют об отсутствии фазового превращения при $200-225^{\circ} \mathrm{C}$. Обнаруженные аномалии электрических характеристик могут быть связаны с высокой подвижностью атомов кислорода в пограничных слоях композита, а не со структурными изменениями и фазовыми переходами в титанате кальция-меди.

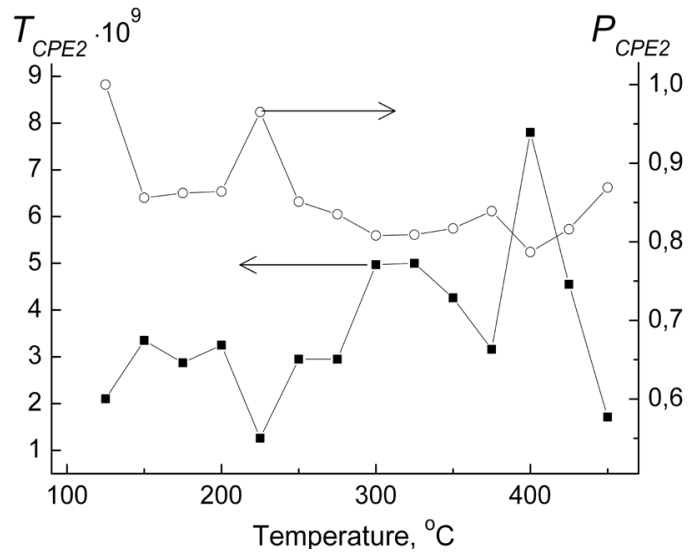

b

Pис. 6. Температурные зависимости параметров $T_{C P E 1}$ и CPE1 (a), $T_{C P E 2}$ и CPE2 (b).

Fig. 6. Temperature dependencies of the parameters of the equivalent circuits $T_{C P E 1}$ and CPE1 (a); $T_{C P E 2}$ and CPE2 (b). 


\section{4. Выводы}

На основании анализа данных, полученных методом импеданс-спектроскопии, показано, что $\mathrm{CaCu}_{3} \mathrm{Ti}_{4} \mathrm{O}_{12}-\mathrm{CuO}$ является материалом со смешанной электронно-ионной проводимостью. Сильная дисперсия проводимости при комнатной температуре объяснена участием в поляризационном процесс ионов меди или протонов, которые могут присутствовать в образце вследствие диссоциативной адсорбции молекул воды из воздуха. При температуре около $300^{\circ} \mathrm{C}$ обнаружен минимум дисперсии диэлектрической проницаемости, что объяснено появлением кислородной проводимости, которая в значительной степени нейтрализует катионную проводимость.

Моделирование электрических свойств методом построения эквивалентных схем показало, что образец можно рассматривать состоящим из двух слоев. Высокоомный слой был отнесен к кристаллической части образца, а низкоомный - к межзеренной прослойке.

Установлено существенное изменение электрических свойств образца при температуре $200-225^{\circ} \mathrm{C}$, несвязанное с фазовым переходом в титанате кальция-меди.

Дополнительный материал/Supplementary Material. Электронная версия статьи содержит дополнительный материал (рисунки и таблииу), доступный безвозмездно на сайте журнала (lettersonmaterials.com)./The online version of this paper contains supplementary material (figures and table) available free of charge at the journal's Web site (lettersonmaterials.com).

\section{Литература/References}

1. H.E. Kim, S.-D. Yang, J.-W. Lee, H.M. Park, S.-I.Yoo. J. Cryst. Growth. 408, 60 (2014). DOI: $10.1016 /$ j.jcrysgro.2014.09.013

2. D. Qin, G. Liang. Alloys and Comp. 549, 11 (2013). DOI: $10.1016 /$ j.jallcom.2012.09.083

3. A. Deschanvres, B. Raveau, F. Tollemer. Bull. Soc. Chim. Fr., 4077 (1967).

4. M.A. Subramanian, D. Li, N. Duan, B.A. Reisner, A. W. Sleight. J.Sol. State. Chem. 151, 323 (2000). DOI: $10.1006 /$ jssc. 2000.8703

5. S. M. Moussa, B. J. Kennedy. Mater. Res. Bull. 36, 2525 (2001). DOI: 10.1016/80025-5408(01)00732-2

6. W. Hao, J. Zhang. Alloys and Comp. 559, 16 (2013). DOI: 10.1016/j.jallcom.2013.01.042

7. C.-M. Wang, K.-S. Kao, S.-Y. Lin, Y.-C. Chen, S.-C. Weng. Phys. Chem. Sol. 69, 608 (2008). DOI: $10.1016 /$ j.jpcs.2007.07.049
8. C.Chen, C. Wang, T.Ning, H.Lu, Y.Zhou, H.Ming, P.Wang, D. Zhang, G. Yang. Sol. St. Comm. 151, 1336 (2011). DOI: $10.1016 /$ j.ssc.2011.06.024

9. J. J. Mohamed, S.D. Hutagalung, M.F. Ain, K. Deraman, Z.A. Ahmad. Mater. Lett. 61, 1835 (2007). DOI: 10.1016/j.matlet.2006.07.192

10. L. Li, Z.W. Wang, X.M. Chen. Mater. Res. Bull. 67, 251 (2015). DOI:10.1016/j.materresbull.2015.01.013

11. B. Wang, Y.-P.Pu, H.-D. Wu, K. Chen, N. Xu. Ceram. Intern. 39, 525 (2013). DOI: 10.1016/j.ceramint.2012.10.127

12. M.V. Gorev, I.N. Flerov, A.V. Kartashev, S. Guillemet-Fritsch. Phys. Sol. St. 54, 1785 (2012). DOI:10.1134/S1063783412090120

13. Z. Yang, Y. Zhang, R. Xiong, J.S. Z. Yang, Y. Zhang, R. Xiong, J.Shi. Mater. Res. Bull. 48, 310 (2013). DOI: 10.1016/j.materresbull.2012.10.029

14. W. Wan, C. Liu, H. Sun, Z. Luo, W.-X. Yuan, H. Wu, T.Qiu. J. Eur. Ceram. Soc. 35, 3529 (2015). DOI: 10.1016/j.jeurceramsoc.2015.05.034

15. W.X. Yuan, S. K. Hark, W.N. Mei. J. Electrochem. 157, 117 (2010). DOI:10.1149/1.3353040

16. J. W. Lee, J.-H. Koh. Ceram. Intern. 41, 10442 (2015). DOI: 10.1016/j.ceramint.2015.04.109

17. P. Liu, Y. Lai, Y. Zeng, S. Wu, Z. Huang, J.Han. Alloys Comp.650, 59 (2015).DOI: 10.1016/j.jallcom.2015.07.247

18. M. Li, X.L. Chen, D.F. Zhang, Q. Liu, C.X. Li. Ceram. Intern. 41, 14854 (2015). DOI: 10.1016/j.ceramint.2015.08.019

19. X. Ouyang, S. Huang, W. Zhang, P. Cao, Z. Huang, W.Gao. J. Sol.St. Chem. 211, 58 (2014). DOI: 10.1016/j.jssc.2013.12.009

20. Y. Li, P. Liang, X. Chao, Z. Yang. Ceram. Intern. 39, 7879 (2013). DOI:10.1016/j.ceramint.2013.03.049

21. L. Sun, Z. Wang, Y. Shi, E. Cao, Y. Zhang, H. Peng, L. Ju. Ceram. Intern. 41, 13486 (2015). DOI: 10.1016/j.ceramint.2015.07.140

22. R. Löhnert, R. Schmidt, J. Töpfer. J. Electroceram. 34, 241 (2015). DOI: 10.1007/s10832-015-9982-0

23. L. G. Akselrud, Yu. N. Grin, P. Yu. Zavalij, et al, CSDuniversal program package for single crystal or powder structure data treatment. Thes. Rep. XII Eur. Crystallogr. Meet. 1985. p. 155.

24. R.E. Newnham, D.P. Skinner and L.E. Cross. Mat.Res. Bull. V. 13, 525 (1978). DOI: 10.1016/0025-5408(78)90161-7

25. N.A. Sekushin, M.S. Koroleva, I.V. Piir. Russ. J. Electrochem. 52, 1032 (2016).

26. N.A. Sekushin, N.A. Zhuk, E.A. Belyaeva et al. Lett. on Mater. 7, 393 (2017). DOI: 10.22226/2410-3535-2017-4-393-397

27. A. G. Krasnov, I. V. Piir, M.S. Koroleva et al. Sol. St. Ion. 302, 118 (2017). 